\title{
Das Presseapostolat nach den Akten des Provinzialkonzils von Guadalajara (1896-1897). Eine Dokumentation.
}

\author{
von Willi Henkel
}

\section{Zur Situation der Kirche in Mexiko}

Seit der Unabhängigkeit Mexikos am 27. September 1821 hatte sich das Verhältnis von Kirche und Staat grundlegend gewandelt. Es kam zur Etablierung einer liberalen, antikirchlichen Republik. Auch nach dem Scheitern des habsburgschen Kaisers Maximilian, der 1867 erschossen wurde, verschlechterte sich die Lage der Kirche noch mehr. Erst während der langen Amtszeit des Präsidenten Porfirio Diaz von 1876-1911, die nur während der Jahre 1880-1884 unterbrochen wurde, kam es zu der sogenannten „Polîtica de Conciliación“, obwohl dieser gleichzeitig auf die ihm notwendig erscheinende Distanz zur Kirche achtete. ${ }^{1}$ Aufgrund der fast 20 Jahre dauernden Freundschaft mit dem Präsidenten glaubte Erzbischof Gillow von Antequera (Oaxaca), daß es möglich sei, nach der 120jährigen Unterbrechung - das vierte mexikanische Provinzialkonzil fand 1771 statt - eine Provinzialsynode einzuberufen, ohne daß man von seiten des Staates mit Schwierigkeiten rechnen müßte. ${ }^{2}$ Das Provinzialkonzil von Antequera fand während der Jahre 1892-1893 in Oaxaca statt. Nach diesem Vorbild fanden bald weitere Provinzialsynoden in den verschiedenen Kirchenprovinzen Mexikos statt: das fünfte mexikanische Provinzialkonzil in der Hauptstadt Mexiko (1896), das erste Provinzialkonzil von Durango (1896), das erste Provinzialkonzil von Guadalajara (1896-1897) und das erste Provinzialkonzil von Michoacán (Morelia). ${ }^{3}$

\section{Das Presseapostolat im Gesamtrahmen des Provinzialkonzils von Guadalajara ${ }^{4}$}

Während die genannten Provizialkonzilien das Presseapostolat kaum oder nur kurz erwähnen ${ }^{5}$, widmete die Provinzialsynode von Guadalajara diesem eine ausführliche Darstellung. Sie erfolgte im vierten und letzten Kapitel über die Ausbreitung des Glaubens nach der Behandlung der verschiedenen Formen des Dienstes am Wort und nach den Erklärungen über die katholischen Vereine. ${ }^{6}$ In 33 Dekreten, die in eine Einleitung und zwei Paragraphen aufgegliedert wurden, erläuterten die Bischöfe ihre Vorstellungen und Anregungen für das Presseapostolat.

\subsection{Die Einleitung}

Der Grund, weshalb die Bischöfe das Thema so ausführlich behandelten, wird in der Einleitung deutlich. Sie betrachten die Verbreitung von guten Schriften als eine pastorale Pflicht. Darüber hinaus würdigen sie in der Einführung die enorme Bedeutung der Presse für das Zustandekommen der öffentlichen Meinung. ${ }^{7}$

Die Ausführungen der Bischöfe über die Angriffe der Presse auf Glauben und Sitten des Volkes müssen mit Bezugnahme auf die antikatholischen Zeitungen der Vergangenheit gelesen werden. Es blieb nicht bei einer Abwertung katholischer Zeitungen, die die Gegner als „kon-

Dr. Willi Henkel omi ist Professor für Missionswissenschaft an der Päpstlichen Universität Urbaniana und Direktor der Bibliothek der Kongregation für die Evangelisation der Völker (Propaganda) in Rom. 
servativ" bezeichneten, sondern es kam zu ernsthaften Auseinandersetzungen. Die ersten katholischen Zeitungen „El Tiempo“ (1846) und „El Universal“ (1848-1854) wurden im Kamp1 mit den Liberalen gegründet. ${ }^{8}$ Während der Revolution von Ayutla wurden die Redaktion und die Druckerei des „Universal“" geplündert und in Brand gesetzt. Ihre Nachfolger waren „La Cruz“ (1855-1858) und „Diario de Avisos“" (1856-1860), dessen Herausgeber D. Vicente Segura Argüelles ermordet wurde. Auch die Zeitung „Pájaro Verde“ (1861, 1863-1877) wurde geplündert. ${ }^{9}$ Unter dem Präsidenten Diaz blühten zahlreiche antikatholische Zeitungen, zu welchen die katholischen Zeitungen „La Voz de México“ (1870-1909) und „El Tiempo" (1883-1912) ein Gegengewicht schaffen sollten. ${ }^{10}$ Nach der Darlegung der Bischöfe waren dazu noch größere Anstrengungen notwendig.

\subsection{Förderung der katholischen Presse}

Die Schriften Papst Leo's XIII. gaben den Bischöfen der Kirchenprovinz Guadalajara zahl. reiche Anregungen für ihre Ausführungen. Ihrerseits zitierten die Bischöfe die Verlautba. rungen dieses Papstes ausgiebig. Sie verlangten von den Schriftstellern ähnliche Einstel. lungen, wie sie Leo XIII. gefordert hatte. J. Schmidlin faßt die Einstellung dieses Papstes fol. gendermaßen zusammen: „Neben der katholischen Buchliteratur begünstigte er eifrig auch die periodische, schätzte die Presse als Großmacht, nahm ihre Vertreter freundlich auf unc erteilte ihnen gern Interviews. Er empfahl ihnen Eleganz und Mäßigung, unterstützte nach K räften die kirchlichen Tagesblätter und Zeitschriften, ermunterte und belohnte auch bei je der Gelegenheit die katholischen Journalisten und Schriftsteller"'!.

\subsection{Die Notwendigkeit einer katholischen Presse}

Ein langes Zitat aus der Enzyklika Leo’s XIII. „Etsi nos“vom 15. Februar 1882 erläutert den zerstörerischen Einfluß schlechter Bücher und Zeitschriften, die falsche Anklagen und $\mathrm{Haß}$ gegen Papst und Kirche verbreiten. ${ }^{12}$ Die Bischöfe bestätigen die Aussagen des Papstes aufgrund ihrer Erfahrungen in Mexiko. Sie weisen ferner darauf hin, daß sich die damals neuen telegraphischen Medien fast ausschließlich in Händen von Gegnern der Kirche befanden. ${ }^{13}$ Nicht selten verbreiten sie ungenaue und falsche Nachrichten über Papst und Kirche. Auch die Behandlung von Skandalen, von denen noch später die Rede sein wird, gefällt den Bischöfen nicht. Sie sind deshalb der Meinung, man müsse als Gegengewicht eine katholische Presse schaffen, die einen wichtigen Beitrag zu einer gesunden Meinungsbildung leisten könne. ${ }^{14}$

\subsection{Der Beitrag von Priestern und Laien}

Priester und Laien können einen wertvollen Beitrag zur Verbreitung guter Literatur leisten Die Bischöfe empfehlen die Unterstützung von katholischen Verlagen und Schriftstellern Sie begründen ihren Aufruf, gute Literatur zu verbreiten, mit mehreren Zitaten aus de Heiligen Schrift ${ }^{15}$, nach denen der Herr jene belohnt, die ihren Beitrag zur Belehrung de Nächsten leisten. Man solle durch die Unterstützung katholischer Schriftsteller den Circu lus vitiosus durchbrechen, der darin bestehe, daß gute Literatur keinen Absatz findet, weil e: keine guten Schriftsteller gebe und es gebe andererseits keine fähigen Schriftsteller, weil e keine Nachfrage gebe. Gute Schriftsteller und Journalisten sollten einen ihren An strengungen entsprechenden Lohn erhalten. 
Auf Einzelheiten, wie Fonds zur Unterstützung von Verlegern und Schriftstellern zu beschaffen sind, gehen die Bischöfe nicht ein. Sie überlassen die Regelung dem einzelnen Bischof. ${ }^{16}$ Dabei sollten bereits bestehende katholische Verlage und Vereine tatkräftig gefördert werden. Wenigstens in den Bischofsstädten sollten diese durch eine Niederlassung vertreten sein.

\subsection{Die Gründung von katholischen Wochenzeitungen}

Besondere Beachtung verdient der modern klingende Vorschlag der Bischöfe, katholische Wochenzeitungen zu gründen ${ }^{17}$, die sogar kostenlos verteilt werden sollten. Im Hinblick auf die so entstehenden Unkosten, die gedeckt werden müssen, erscheint dieser Plan allzu optimistisch. Realistischer ist der Hinweis der Bischöfe auf die zahlreichen Möglichkeiten, von denen Priester, Lehrer und Laien Gebrauch machen können, um gute Literatur an den Leser zu bringen.

\subsection{Ausbildungsstätten für Journalisten}

Aufgeschlossen klingt auch der Plan, in jeder Diözese der Kirchenprovinz, oder wenigstens in der Erzdiözese Guadalajara, Journalistenschulen zu gründen.18 Für diese Institute nannten die Bischöfe die Priesterseminarien als den geeigneten Ort. Dazu ist zu beachten, daß damals auch Laien in den Priesterseminarien von Mexiko und Guadalajara zusammen mit den Priesteramtskandidaten Philosophie, Jurisprudenz und Geschichte studierten. ${ }^{19}$ Neben diesen Fachrichtungen sollten die angehenden Journalisten vor allem in der Kunst des Schreibens ausgebildet werden.

\subsection{Orientierung für katholische Schriftsteller}

Wie bereits oben erwähnt wurde, waren es pastorale Erwägungen, die die Bischöfe bewogen, sich zum Presseapostolat zu äußern. Sie betonen eigens, daß sie nicht die freie Meinung der Schriftsteller und Journalisten einengen möchten. ${ }^{20}$ Vielmehr möchten sie diese in ihrer verantwortungsvollen Arbeit ermuntern. Als Beweggrund dafür nennen sie den überaus wertvollen Beitrag der Schriftsteller für den Aufbau des Leibes Christi, der Kirche. Deshalb sollen diese die Freiheit und die Rechte der Kirche ebenso wie ihre Lehren auf redliche Weise verteidigen und erklären. Die Bischöfe haben aus der Geschichte ihres eigenen Landes gelernt, daß man unnötige Streitereien vermeiden sollte. Deshalb empfehlen die Bischöfe den Schriftstellern und Journalisten eine positive Haltung, wie sie der hl. Franz von Sales gelehrt hat. ${ }^{21}$ Dieser vertritt die Ansicht, daß der Schriftsteller die Wahrheit mit Liebe darlegen soll. Er muß Respekt vor der Meinung der anderen haben. Für die Lehren der Kirche sollte er ein sicheres Gespür haben, was vermeiden hilft, daß er das Gegenteil kirchlicher Lehre insinuiert. Nach der Meinung der Bischöfe sollten kontroverse Fragen unter Fachleuten und Gelehrten ausgetragen werden und erst nach ihrer Klärung dem Volk vorgetragen werden.

Wie Papst Leo XIII. schlagen die Bischöfe vor, die Schriftsteller sollten den hl. Thomas als Vorbild betrachten. ${ }^{22}$ Dieser bleibt auch bei der Verteidigung des Glaubens stets maßvoll im Umgang mit Andersdenkenden und Gegnern.

Originell scheinen die Ausführungen über die Nächstenliebe, wie Schriftsteller und Journalisten diese in ihrem Beruf leben und verwirklichen können. ${ }^{23}$ Hier ist die Bezugnahme auf die Ausführungen des Apostels Paulus über die Liebe im 13. Kapitel des ersten Korinther- 
briefes eindeutig. Der Schriftsteller und Journalist, der die christliche Liebe als Maßstab nimmt, begünstigt nicht den Irrtum, sondern sucht stets die Wahrheit mitzuteilen. Er hat das Wohl der Kirche und das Heil seiner Brüder im Auge. Wenn er für die Wahrheit kämpft, dann tut er es ohne Stolz und Anmaßung, sondern mit christlicher Bescheidenheit. Es ist mehr eine Belehrung als ein Kampf um einen Sieg. Die Aufrichtigkeit verlangt von ihm, daß er nichts Ungewisses oder Unbekanntes bestätigt. Wer von der christlichen Liebe beseelt ist, glaubt an das Gute im Menschen.

Die Bischöfe leiten die Maßstäbe für das Mitteilen und Behandeln von Skandalen aus der christlichen Liebe ab, für den Fall, daß diese überhaupt mitgeteilt werden müssen. Ihre Darstellung darf den Glauben und die Sitten der Gläubigen nicht in Gefahr bringen. ${ }^{24}$ Schließlich sollten Schriftsteller und Journalisten alle Härten und Bitterkeiten in ihren Schriften meiden.

Die Erfüllung dieser Aufgaben verlangt von den Schriftstellern ein ernsthaftes Studium und das Streben nach Wahrheit, Demut und Gerechtigkeit. Die Bischöfe schätzen den Einsatz der Schriftsteller und Journalisten als Dienst für Kirche und Vaterland hoch ein ${ }^{25}$ und ermuntern diese, diesen im Sinne der Kirche auszuüben.

Die Darlegungen der Bischöfe und die zahlreichen Zitate Papst Leo's XIII. lassen deutlich erkennen, wie sehr diese sich am päpstlichen Magisterium orientiert haben und sich von ihm inspirieren ließen. Von den Vorschlägen verdienen vor allem die Gründung katholischer Wochenblätter ${ }^{26}$ und die journalistischen Ausbildungsstätten besondere Beachtung. Das Ethos, das ihren Richtlinien zugrunde liegt, behält in seinen Grundzügen auch heute noch seine Gültigkeit, obwohl sich seit jener Zeit die Lebensumstände beträchtlich gewandelt haben.

\section{Anmerkungen}

1 H.J. Prien: Die Geschichte des Christentums in Lateinamerika. Göttingen 1978. $725 \mathrm{f}$.

2 Nicht paginierter Brief von Erzbisch of Gillow an den Präfekten der Konzilskongregation in Rom (heute Kleruskongregation genannt) Kardinal Aloysius Serafini vom 28. August 1893. Dieser befindet sich im Archiv dieser Kongregation, in dem die Provinzialkonzilien in einem eigenen Raum aufbewahrt werden. Dort wird auch die im folgenden verwandte Dokumentation aufbewahrt.

$3 \mathrm{Vgl}$. dazu: Acta et Decreta Concilii Mexicani Quinti celebrati anno Domini 1896 Metropolita Ill.mo ac Rev.mo Prospero Maria Alarcón y Sánchez de la Barquera. Typis Vaticanis. Romae 1898. Die Manuskripte befinden sich in der Position ACC (= Archiv der Kleruskongregation) 55. - Concilium Provinciale Durangense Primum 1896: ACC 30. Es handelt sich um das Manuskript und um den vorläufigen Druck. - Acta et Decreta Concilii Provincialis Guadalaxarensis Primi anni Domini 1896 et 1897 Guadalaxarae celebrati. Typis Vaticanis, Romae 1905. ACC 36. - Acta et Decreta Concilii Provincialis Mechoacanesis Primi anno Domini 1897. Typis Vaticanis, Romae 1905. ACC 53.

4 Die Teilnehmer waren: Erzbischof Pedro Loza y Pardavé von Guadalajara, Bischof Buenaventura Portillo y Tejeda von Zacatecas, Atenogenes Silva Valvarez Tostado von Colima und Bischof Ignacio Diaz Macedo von Tepic. Sekretär des Provinzialkonzils war Florencio Parga. Wie üblich nahmen auch Theologen, Kirchenrechtler und weitere Fachleute an den Beratungen teil.

5 Vgl. z. B. Acta et Decreta Concilii Mexicani Quinti, 73-74.

6 Acta et Decreta, 57-67.

7 Acta et Decreta, 57f.

8 Vgl. W. Guinea: Catholic Press, Mexico, in: New Catholic Encyclopedia IIl, 307-308.

9 W. Guinea, a.a.O., 308.

10 W. Guinea, a.a.O., 308.

I1 J. Schmidlin: Papstgeschichte der Neuesten Zeit. Bd. II: Papsttum und Päpste gegenüber den modernen Strömungen, Pius IX. und Leo XIII. (1846-1903) München 1934, 391. Schmidlin gibt dort auch die wichtigste Literatur zu diesem Thema an.

12 Acta et Decreta, 58-59. Die Enzyklika „Etsi nos“ kann gefunden werden in: Acta Leonis XIII vol.III (1884), 13-27. Sie war ursprünglich an die italienischen Bischöfe gerichtet.

13 Acta et Decreta, 66. 
14 Uber die politischen Ideen der Presse und ihre kämpferische Einstellung vgl. M.C.R.C. (Marîa del Carmen Ruiz Castañeda), Periodismo, in: Enciclopedia de México X (México 1977), 222-257.

15 Acta et Decreta, 61.

16 Acta et Decreta, 62f.

17 Acta et Decreta, 62.

18 Acta et Decreta, 62.

19 Acta et Decreta, 62. - Zu den Seminarien vgl. J.H. Lee: Clerical education in Nineteenth-Century Mexico: The Conciliar Seminaries of Mexico City and Guadalajara, 1821-1910, in: The Americas 36 (Washington D.C. 1980), 465-477.

20 Acta et Decreta, 63.

21 Acta et Decreta, 64.

22 Acta et Decreta, 64.

23 Acta et Decreta, 65.

24 Acta et Decreta, $65 f$.

25 Acta et Decreta, 66.

26 Die Anzahl der katholischen Zeitungen und Bistumsblätter nahm in diesem Jahrhundert zu. Nach der Mitte des Jahrhunderts besaß jede Diözese ihre Kirchenzeitung, einige Diözesen gaben eine solche gemeinsam heraus. $\mathrm{Vgl}$. W. Guinea, a.a.O., 308.

\section{Anhang}

\section{Caput IV. \\ De catholico prelo.}

136. Cum in dies crescere videamus in Ditione nostra tum scribendi licentiam, tum insatiabilem aviditatem legendi, ita ut ex hoc librorum et ephemeridum scriptores domini vere videantur opinionis publicae, eamque in quoscumque errores adducere valeant, et reapse magna ex hac parte adducant, et hic, uti in caeteris, valde sit deplorandum filios huius saeculi prudentiores esse filiis lucis; et quia ex debito pastoralis officii nihil ex parte nostra praetermittendum est, ut libri salubres, sanae doctrinae, et qui vere prosint legentibus, propagentur et disseminentur inter fideles nobis commissos, tanto maiori studio quo magis in dies augentur conatus et prope incredibilis diligentia haereticorum et sectariorum hominum, qui tempus, ingenium, pecunias et labores eo conferunt, ut detestabiles contra Deum, sanctam Ecclesiam, catholicam fidem et honestos mores libellos pene infinitos ubique locorum palam vel subdole introducant, ut quod diabolus insidiis suis, artibusque nondum est assecutus, ipsi libellis suis perficiant, animasque catholicorum, fide deleta, moribusque corruptis, in perpetuum interitum adducant: Synodus haec Provincialis, tum ad prelum catholicum in Provincia fovendum, tum ut ipsum prelum facilius recto tramite semper incedat, quae sequuntur statuit:

\section{Kapitel: Die katholische Presse}

136. Tagtäglich sehen wir, wie in unserer Region sowohl die Freizügigkeit im Schreiben zunimmt als auch die unersättliche Begierde zu lesen, so daß deshalb die Verfasser von Büchern und die Journalisten als die Herren der öffentlichen Meinung erscheinen und diese in alle möglichen Irrtümer zu führen vermögen und sie tatsächlich auch weitgehend dahin führen; dabei ist, wie auch sonst, sehr zu bedauern, daß die Kinder dieser Welt klüger sind als die Kinder des Lichtes. Andererseits dürfen wir aufgrund der Verpflichtung unseres Hirtenamtes nichts unterlassen, daß heilsame Bücher mit gesunder Lehre, die für die Leser wirklich von Nutzen sind, möglichst weit unter den uns anvertrauten Gläubigen verbreitet werden, und das um so eifriger, je mehr von Tag zu Tag die Versuche und die geradezu unglaubliche Beflissenheit der Irrlehrer und Sektierer zunehmen, die Zeit und Geist, Geld und Arbeit dafür einsetzen, abscheuliche und fast unzählige Schriften gegen Gott und die hl. Kirche, gegen den katholischen Glauben und die ehrbaren Sitten allenthalben offen oder heimlich einzuführen; so wollen sie mit ihren Schriften das bewirken, was der Teufel mit seinen Nachstellungen und Verführungsk ünsten noch nicht zustandegebracht hat, und die Seelen der katholischen Chri- 
sten mit der Zerstörung des Glaubens und der Zerrüttung der Sitten in das ewige Verderben stürzen. Um also die katholische Presse in unserer Kirchenprovinz zu fördern und sicherzustellen, daß die katholische Presse jederzeit leichter auf dem rechten Wege voranschreitet, hat diese Provinzialsynode die folgenden Bestimmungen erlassen:

\section{$\S 1$. De prelo catholico fovendo.}

137. Ad prelum catholicum fovendum, ante omnia recogitari ab omnibus iubemus quae S.P. Leo XIII enixe his verbis commendat ${ }^{1}$ ( ${ }^{1}$ Encycl. Etsi nos, 15 Feb. 1882): "Qui capitali odio ab Ecclesia dissident, scriptis editis decertare, iisque tanquam aptissimis ad nocendum armis uti consuevernunt. Hinc teterrima librorum colluvies, hinc turbulentae et iniquae ephemerides, quarum vesanos impetus nec leges frenant, nec verecundia continet. Quidquid proximis his annis per seditiones et turbas gestum est, iure gestum esse defendunt; dissimulant aut adulterant verum; Ecclesiam et Pontificem quotidianis maledictis falsisque criminationibus hostiliter petunt, nec ullae sunt tam absurdae pestiferaeque opiniones, quas non disseminare passim aggrediantur. Propterea scripta scriptis opponenda sunt, ut res quae potest plurimum ad perniciem, eadem ad hominum salutem et beneficium transferatur, et inde remedia suppetant, unde mala venena quaeruntur ». Dein quae sequuntur praecipimus:

138. Quoniam ephemerides quotidiano suo commercio una cum rerum novarum nuntiis animos doctrinis variis imbuunt et quasi satiando implent, summa cura adhibeatur, ut, eliminatis falsis et perniciosis foliis, illa potius habeantur et legantur, quae ad veritatem pietatemque parant. Eo sane modo etiam implebuntur vota Sanctissimi Domini nostri Leonis XIII, cum ad Episcopos Lusitaniae, similibus in adiunctis ver-

\section{$\S 1$. Die Förderung der katholischen Presse}

137. Zur Förderung der katholischen Presse sollen alle an erster Stelle wieder bedenken, was Papst Leo XIII. eindringlich mit folgenden Worten empfiehlt: „Diejenigen, die der Kirche mit tödlichem $\mathrm{Haß}$ entgegenstehen, pflegen ihren Kampf mit der Herausgabe von Schriften zu führen und diese als höchst geeignete Waffen zu gebrauchen, um Schaden zuzufügen. Daher die garstige Flut von Büchern, daher die wirren und feindlichen Zeitungen, deren wahnsinnige Angriffe weder Gesetze hemmen noch die Scham in Schranken hält. Was immer in diesen letzten Jahren durch Aufstände und Unruhen getan wurde, verteidigen sie als zu Recht getan; sie verschweigen oder verfälschen die Wahrheit; Kirche und Papst greifen sie mit täglichen Schmähungen und falschen Beschuldigungen an; auch gibt es keine noch so törichten und verderblichen Meinungen, die sie nicht allenthalben zu verbreiten suchen. Deshalb müssen Schriften gegen Schriften gesetzt werden, damit dasselbe, das sehr viel zum Verderben vermag, zum Heil und zur Wohltat für die Menschen übernommen werde, und die Heilmittel von daher zu Hilfe kommen, von wo die üblen Gifte geholt werden." Deshalb verordnen wir das Folgende:

138. Weil die Zeitungen bei ihrem täglichen Vertrieb zugleich mit den Meldungen von Neuigkeiten die Geister mit allerlei Lehren anfüllen und gleichsam sättigen, ist äußerste Mühe anzuwenden, daß die falschen und verderblichen Blätter ausgemerzt und eher solche gehalten und gelesen werden, die zur Wahrheit und Frömmigkeit hinfuhren. Auf diese Weise werden auch Wunsch und Verlangen unseres Heiligen Vaters Leo's XIII. erfüllt, wie er kürzlich an die Bischöfe von 
santes nuper scribebat et animadvertebat: "Plurimum iuverit, si cura ductuque vestro diaria publicentur, quae malis venenis undeumque oblatis opportune medeantur, suscepto veritatis religionis patrocinio ".

139. Optand um foret ut tam fidelium, quam sacerdotum zelus studiaque concurrerent ad erigendas in variis paroeciis, caute tamen et prudenter, bibliothecas ad mutuandos libros, opuscula, et ephemerides; unde omnes absque periculo salutis, legendi cupiditati, quae omnes ubique invadit, possent facere satis per scripta vere utilia, tum de sacris et religiosis, tum de profanis rebus, et ad honeste relaxandos quoque animos, vel gratis vel exiguo pretio eis tradita.

140. In aliis locis et oppidis, ubi id expedire visum fuerit, aliquem pium designare virum oportet, qui cum catholicis editoribus relationem instituens, mediator sit inter eos et loci fideles ad praefatos libros et opuscula et folia prompte et commode habenda.

141. Catholicos vero divites hortamur in Domino ut subsidia praebeant, ad promovendum scripta catholica et, speciali modo, ob necessitatem praesentem, ephemerides bonas, quo melius hac in re ipsis notitiis superare improbas possint.

142. Eos insuper viros sive clericos sive laicos, qui necessariis dotibus pollent, in Domino provocamus et invitamus, ut quocumque potuerint modo, sive scribendo, sive adiuvando scriptores cum opportunis notitiis, in propugnationem fidei et morum honestatis vires impendant, scientes optimum et dignissimum opus esse invictae veritatis praeconium. Praefata christiani fervoris conamina optimum, coniuncta plurium operatione, Deo largiente, effectum sortientur.
Portugal, die sich in ähnlicher Lage befinden, geschrieben und vermerkt hat: „Von größtem Nützen wird es sein, wenn dank Eurer Mühe und Leitung Tageszeitungen veröffentlicht werden, die gegen die von allen Seiten angebotenen verderblichen Gifte Abhilfe schaffen, unter dem Schutz und Schirm der Wahrheit und der Religion".

139. Es wäre zu wïnschen, daß der Eifer und die Bemühungen der Priester und der Gläubigen sich zusammentäten, um in den verschiedenen Pfarreien, vorsichtig und klug natürlich, Bibliotheken zu errichten zum Ausleihen von Büchern, Kleinschriften und Zeitungen. So könnten alle ohne Gefahr für das Heil ihre Leselust, die alle überall befällt, befriedigen, und das mittels wahrhaft nützlicher Schriften, - sowohl über heilige und religiöse als auch über profane Gegenstände, auch zum Zweck einer ehrbaren Geistesentspannung, - die ihnen entweder kostenlos oder gegen geringes Entgelt überlassen werden.

140. An anderen Orten und in Städten, wo es nützlich erscheinen sollte, muß man einen gottesfürchtigen Mann bestimmen, der die Verbindung mit den katholischen Verlegern aufnimmt und zwischen diesen und den Gläubigen des Ortes als Vermittler auftritt, damit man schnell und leicht in den Besitz der vorher genannten Bücher, Kleinschriften und Zeitungen kommt.

141. Die wohlhabenden Katholiken aber ermahnen wir im Herrn, die Hilfsmittel zur Förderung des katholischen Schrifttums zu spenden, und vor allem, wegen der augenblicklichen Notwendigkeit, für gute Zeitungen, damit sie auf diesem Gebiet mit ihren eigenen Nachrichten die schlechteren übertreffen können.

142. Außerdem rufen wir Kleriker und Laien, die über die nötige Begabung verfügen, im Herrn auf und laden sie ein, auf jede nur mögliche Weise - sei es mit Schreiben, sei es mit Besorgen von geeigneten Nachrichten für die Schriftsteller - ihre Kräfte für die Verteidigung des Glaubens und der Ehrbarkeit der Sitten einzusetzen in dem Bewußtsein, daß die Verherrlichung der unbesiegbaren Wahrheit ein sehr gutes und geziemendes Werk ist. Solche Unternehmungen christlichen Eifers werden dank der Zusam- 
143. Parochis et Sacramenti Poenitentiae ministris, enixe commendamus, ut nihil omittant, ut boni ac salutares libri, qui non modo pietati et instructioni, sed et honestae etiam relaxationi absque ullo saluti periculo inserviant, fidelibus prudenter indicent et commendent.

144. Instituendis ephemeridibus catholicis, iis maxime quae dominicales vocantur paginae, et bonis libris in usum populi edendis et mutuandis, visum est nobis, laicorum societates perutiles esse et $a b$ Episcopis quam studiosissime adiuvari debere, ad earum regimen opportunis statutis normis.

145. Pro iuventute vero libri comparentur, non phantasiae, etiamsi pii videantur, nimium indulgentes, sed tenoris potius historici, vel qui aliis modis salubriter instruunt; simul autem continue vigilandum, ne nimis frequenti librorum porrectione periculosus legendi pruritus excitetur.

146. Omnes tum pietate, tum doctrina praestantes huius Ecclesiasticae Provinciae viros, et praesertim sacerdotes, obsecramus in Domino, ut, contraria contrariis curando, parvae molis scripta, ab Episcopis antea probata, edant in aedificationem fidei, ac salutarem populi instructionem ac, ut eadem scripta, uti et alii incorruptae pariter doctrinae probateaque utilitatis libri ab aliis conscripti, prout locorum aut personarum ratio suggesserit, inter fideles diffundant.

147. Quonıam vero non desunt Patria in nostra societates et typographi editores, qui evulgatione libellorum sanae doctrinae zelantes adlaborant, eosque commodo pretio dant; ut ab omnibus facile comparari queant, illos magnopere commendamus, Parochi autem caeterique sacerdotes fideles adhortentur ut libros, opuscula, et folia, menarbeit von vielen mit Gottes Hilfe besten Erfolg haben.

143. Den Pfarrern und den Verwaltern des Bußsakramentes empfehlen wir angelegentlich, nicht zu versäumen, den Gläubigen in kluger Weise gute und heilsame Bücher anzugeben und zu empfehlen, die nicht nur der Frömmigkeit und der Belehrung dienlich sind, sondern auch der ehrbaren Entspannung, ohne irgendeine Gefahr für ihr Seelenheil.

144. Zur Gründung von katholischen Zeitungen, und besonders von sogenannten Sonntagsblättern, sowie zur Herausgabe und Ausleihe von guten Büchern für den Gebrauch der Leute halten wir die Vereinigungen von Laien für sehr nützlich; diese sollen deshalb von den Bischöfen durch das Erlassen von geeigneten Vorschriften für ihre Leitung auf das eifrigste unterstützt werden.

145. Für die Jugend sollen Bücher bereitgestellt werden, die, auch wenn sie fromm sein sollten, nicht zu sehr der Phantasie huldigen, sondern eher Geschichtliches beinhalten, oder solche, die auf andere Weise heilsam belehren. Zugleich aber muß beharrlich darüber gewacht werden, daß nicht durch zu häufige Bücherausgabe ein gefährlicher Lesereiz geweckt wird.

146. Alle durch Frömmigkeit und Gelehrsamkeit hervorragenden Männer dieser Kirchenprovinz, und vor allem die Geistlichen, beschwören wir im Herrn, daß sie - Gegensätzliches mit Gegensätzlichem zu heilen $K$ leinschriften, die vorher von den Bischöfen gebilligt worden sind, zur Erbauung und heilsamen Belehrung des Volkes veröffentlichen; auch sollen sie andere Bücher von gleichfalls unverdorbenem Lehrinhalt und bewährtem Nutzen, die von anderen verfaßt wurden, unter den Gläubigen verbreiten, wie es die Verhältnisse der Orte und der Personen nahelegen mögen.

147. Weil es aber in unserem Vaterland nicht an Gesellschaften und Verlegern fehlt, die sich mit Eifer der Verbreitung von Schriften gesunder Lehre widmen und sie zu günstigen Preisen abgeben, daß sie von allen leicht erworben werden können, empfehlen wir diese angelegentlich. Die Pfarrer aber und die übrigen Priester sollen die Gläubigen er- 
quae ab illis eduntur, comparare studeant.

148. Eos insuper viros sive clericos sive laicos, qui facultatibus necessariis pollent, in Domino provocamus et invitamus, ut, sive adiuvando scriptores et editores, sive typographias catholicas stabiliendo, praesertim in civitatibus episcopalibus, sive quotquot possint, in propugnationem fidei et morum honestat is vires, ingenium ac pecuniam impendant; scientes magnam benedictionum segetem sibi reservatam haberi a Domino dicente: "Qui ad iustitiam erudiunt multos, fulgebunt quasi stellae in perpetuas aeternitates" (Dan. XII, 3.).

149. Non minori studio procurandum est in omnibus Provinciae Dioecesibus piam ephemeridem titulo " Hebdomada Catholica ", vel alio simili, in lucem prodi, quae grat is fidelibus distribuatur, in qua ipsi utilia et practica quoad fidem, pietatem, et honestatem inveniant et legant: bonum semen seminabitur, quod, benedicente Domino, fructum afferet uberrimum. Ad hoc sanctum opus perficiendum, caritatem et piam divitum largitatem invocamus eisque dicimus: "Honora Dominum de ma substantia " (Prov. 1II, 9.); " potens est autem Deus omnen gratiam abundare facere in vobis, ut in omnibus semper omnem sufficientiam habentes, abundetis in omne opus bonum " (Cor. IX, 8.)

150. Ut abundantius lux diffundatur, solliciti sint Christi sacerdotes, ut, sive in scholis, sive in catechesis exercitiis, sive in congregationibus, quarum cura ad ipsos pertineat, libri conspicui, folia religiosa et piae imagines praemio sint virtuti et scientiae.

151. Optandum foret, ut cunctis in Dioecesibus, aut saltem in Metropoli nostrae Provinciae, institueretur, in Seminariis et in Studiorum Superiorum Institutis, schola scriptorum ephemeridum, quae ab Ordinario mahnen, sich Bücher, Kleinschriften und Blätter, die von jenen herausgegeben werden, zu besorgen.

148. Darüber hinaus rufen wir diejenigen, die über die notwendigen Mittel verfügen, seien sie Geistliche oder Laien, im Herrn auf und laden sie ein, ihre Kräfte, Geist und Geld für die Verteidigung des Glaubens und der Ehrbarkeit der Sitten einzusetzen, sei es durch die Unterstützung von Schriftstellern und Herausgebern, sei es durch die Gründung von katholischen Druckereien, besonders in den Bischofstädten oder, wodurch immer sie vermögen. Dabei sollen sie wissen, daß ihnen eine reiche Saat von Segnungen vom Herrn bereit gehalten ist, der da sagt: „Die, welche viele zur Gerechtigkeit erziehen, werden leuchten wie die Sterne in alle Ewigkeit“" (Dan 12,3).

149. Mit nicht geringerem Eifer ist dafür zu sorgen, daß in allen Diözesen der Provinz ein frommes Blatt herausgegeben wird unter dem Titel: „Die katholische Woche" oder einem anderen ähnlichen, das kostenlos unter den Gläubigen verteilt wird; darin sollen sie Nützliches und Praktisches zu Glauben, Frömmigkeit und Ehrbarkeit vorfinden und lesen. So wird der gute Samen gesät werden, der mit dem Segen des Herrn reichste Frucht bringen wird. Um dieses heilige Werk zu vollbringen, appellieren wir an die Nächstenliebe und die fromme Freigebigkeit der Reichen und sagen ihnen: „Ehre den Herrn mit deinem Vermögen“" (Spr 3,9). „Gott aber ist mächtig, euch jede Gnade reichlich zukommen zu lassen, auf daß ihr in allem stets volles Auskommen habt und darüber hinaus Möglichkeit zu jedem guten Werke" $(2$ Cor 9,8).

150. Damit das Licht des Glaubens reichlicher verbreitet werde, sollen die Priester Christi dafür sorgen, daß sowohl in den Schulen als auch bei den katechetischen Ubungen oder in den Versammlungen, die sie zu betreuen haben, wertvolle Bücher, religiöse Blätter und fromme Bilder als Preis für Tugend und Wissen dienen.

151. Es wäre zu wünschen, daß in allen Diözesen, oder wenigstens in der Metropole unserer Provinz, in den Seminarien oder den Instituten für höhere Studien eine Schule für Journalisten errichtet würde, die der Wei- 
normam acciperet, ubi nonnulli iuvenes solida pietate insignes et ad hoc nobile munus scriptoris exequendum dotes prae se ferentes, in arte pro Religione perite certandi edocerentur, sub fine tantum studiorum et absque ullo eorumdem studiorum nocumento. Hac in palaestra magistri probati prae oculis haberent, tanquam huius disciplinae basim, monita omnia, quae ad scriptores catholicos directa summis a Pontificibus et a Conciliis emanarunt, et plurimum adiuvarent exempla virorum peritissimorum hoc litium in genere, ac discreta lectio, Revistarum, diariorum et foliorum optimorumm quae in die praeluceant inter huiusmodi publicationes.

152. Ordinarii denique, ad hucusque dicta in praxim reducenda, aptas normas vel praecepta quamprimum prudenter expedient pro creando vel fovendo prelo catholico, considerantes necessitat is huius gravitatem, gratias spirituales fidelibus et Christi militibus in hoc praelio decertantibus concedentes, et excogitantes media opportuna, colligendi necessariam pecuniam ad fundationem et sustentationem huius operis; et nunquam oblivioni tradentes, quod scriptores pauperes, qui in hunc laborem tempus et actionem suam consecrant, libenter etiam remunerari debent, et quod ephemerides, ut stabiliantur saltem per tempus aliquod, necessario egent auxilio pecuniae ad sumptus suos cooperiendos; sic vitabitur circulum vitiosum, id est, quod ephemerides non plures habeant subscriptores, quia non sunt illis optimi redactores, et vicissim quod non habeant scriptores meritissimos, quia subscriptores plurimi non sunt illis.

\section{$\S$ II. De Servandis a Scriptoribus catholicis.}

153. Scriptores catholicos laudantes, qui recto spiritu ducti ac sincero veri et boni amore flagrantes, tanquam Religionis asser- sung des Bischofs unterstände. Darin sollten einige, durch gediegene Frömmigkeit ausgezeichnete und Begabung für diese edle Aufgabe eines Schriftstellers aufweisende junge Männer in der Kunst unterrichtet werden, die Religion geschickt zu verteidigen, jedoch erst gegen Ende der Studien und ohne Nachteil für ihre Studien. In dieser Schule sollten bewährte Lehrer gleichsam als Grundlage für dieses Fach alle Mahnungen vor Augen haben, die von den Päpsten und den Konzilien an die katholischen Schriftsteller erlassen worden sind; von größtem Nutzen wären da auch die Vorbilder von sehr erfahrenen Fachleuten in dieser Kampfweise sowie die sorgsam ausgewählte Lektüre von sehr guten Zeitschriften, Tagesblättern und Zeitungen, die zur Zeit unter Veröffentlichungen dieser Art hervorragen.

152. Schließlich sollen die Ordinarien zur praktischen Verwirklichung des bisher Gesagten so bald wie möglich geeignete Regeln oder Vorschriften zur Schaffung oder Förderung der katholischen Presse mit Klugheit erlassen. Die Wichtigkeit dieser ernsten Angelegenheit bedenkend, sollen sie den Gläubigen und Streitern Christi, die an diesem Kampf teilnehmen, geistliche Gnaden gewähren und geeignete Mittel und Wege ersinnen, das für die Gründung dieses Werkes und Erhaltung notwendige Geld zu sammeln. Dabei sollen sie nie vergessen, daß arme Schriftsteller, die ihre Zeit und Tätigkeit dieser Arbeit widmen, auch bereitwillig $z u$ entlohnen sind, und da $\beta$ die Zeitungen, um wenigstens eine Zeitlang fest zu bestehen, notwendig finanzielle Hilfe brauchen, um ihre Unkosten zu decken. So wird der Zirkelschlu $ß$ vermieden werden, daß die Zeitungen nicht mehr Bezieher haben, weil sie nicht über die besten Redakteure verfügen, und umgekehrt, daß sie nicht die verdienstvollsten Redakteure haben, weil sie nicht sehr viele Bezieher aufweisen.

\section{$\S$ II. Was katholische Schriftsteller beobachten müssen}

153. Während wir die katholischen Schriftsteller loben, die vom rechten Geist geleitet und von aufrichtiger Liebe zum Wahren und 
tores, dogmata fidei, Ecclesiae iura ac libertatem morumque catholicorum sanctitatem vindicare ac tueri conantur, et Ecclesiae ita sunt adiutores ad consummationem sanctorum in aedificationem Corporis Christi, rem opportunam et pastoralis sollicitudinis dignam existimamus, monita quaedam, vi auctoritatis divinae nobis creditae ac in spiritu caritatis, qua omnes in Christo amplectimur, illis tradere; non quidem ea mente, ut scribendi studium comprimatur, vel rescindatur legitima in dubiis opinandilibertas; verum ut via securior pateat, et scopulos undequaque imminentes declinent, in quos nonnulli, minus caute ambulantes, saepe saepius impingunt, atque inde ex eorum scriptis uberiores Ecclesia fructus percipiat. Ad hoc summopere nos allicit adhortatio et exemplum Sanctissimi in Christo Patris et Domini Leonis XIII, qui pluries monita catholicis tam librorum quam ephemeridum scriptoribus, per Episcopos dare dignatus est.

154. Experientia quotidiana compertum est, concertationes non solum de religione, sed etiam de scientiis, de historia ac praecipue de politica in aestuosas et contumeliosas disceptationes saepissimae declinare; unde ut optime ait S. Franciscus Salesius, plus laeditur caritas, quam illustratur veritas. Summo igitur studio vitetur haec decertandi ratio, quae omnino aliena est a spiritu defensorum veritatis, quos dedecet esse contentiosos, immo et discipulorum Christi, qui non est dissensionis Deus, sed pacis. Opportunum itaque censemus in memoriam omnium revocare verba Benedicti XIV: "Cohibeatur scriptorum licentia, qui, ut aiebat Augustinus, sententiam suam amantes, non quia vera est, sed quia sua est, aliorum opiniones non modo improbant, sed illiberaliter etiam notant atque traducunt. Non feratur omnino privatas sententias, veluti certa ac definita Ecclesiae dogmata a quopiam in libris obtrudi, opposita vero erroribus insimulari: quo turbae in Ecclesia excitantur, dissidia inter doctores aut feruntur aut fovent ur, et christianae caritatis vincula persaepe abrumpuntur" .
Guten entbrannt, als Vertreter der Religion die Wahrheiten des Glaubens, die Rechte und die Freiheit der Kirche und die Heiligkeit der katholischen Sitten zu verteidigen und zu schützen versuchen und so Helfer der Kirche sind zur Heranbildung der Heiligen für den Aufbau des Leibes Christi, halten wir es für eine nützliche und unserer Hirtensorge angemessene Sache, ihnen kraft der uns von Gott verliehenen Autorität und im Geiste der Liebe, mit der wir alle in Christus umfangen, einige Ermahnungen zu erteilen. Dies geschieht nicht mit der Absicht, den Eifer zum Schreiben zu dämpfen oder die rechtmäßige Meinungsfreiheit in Zweifelsfällen zu beschneiden, sondern, damit ihnen ein sicherer Weg offensteht und sie die von allen Seiten drohenden Klippen vermeiden, gegen die einige, die weniger vorsichtig vorgehen, öfter anstoßen, und daß die Kirche aus ihren Schriften reichere Früchte empfängt. Dazu spornt uns gar sehr die Ermahnung und das Beispiel unseres Heiligen Vaters in Christus des Papstes Leo's XIII. an, der sich mehrmals gewürdigt hat, den katholischen Schriftstellern und Journalisten durch die Bischöfe Mahnungen zu erteilen.

154. Aus täglicher Erfahrung ist bekannt, daß Dispute nicht nur über Religion, sondern auch über Wissenschaften, über Geschichte und besonders über Politik, sehr oft in hitzige und schmähsüchtige Streitereien ausarten; infolgedessen wird dabei, wie der hl. Franz von Sales sehr treffend sagt, mehr die Liebe verletzt als die Wahrheit erhellt. Deshalb soll mit allem Eifer diese Art von Streit vermieden werden, die durchaus dem Geist der Verteidiger der Wahrheit fremd ist, für die es ungeziemend ist, streitsüchtig zu sein, und sogar auch dem Geist der Jünger Christi, der nicht ein Gott des Streites, sondern des Friedens ist. Daher halten wir es für angebracht, allen die Worte Benedikt XIV ins Gedächtnis zurückzurufen: „Man halte die Zügellosigkeit der Schriftsteller im Zaume, die, wie Augustinus sagte, in ihre Meinung verliebt- nicht weil sie wahr ist, sondern weil es ihre ist - die Meinungen der anderen nicht nur mißbilligen, sondern auch in unfairer Weise anführen und übertragen. Man dulde keineswegs, daß persönliche Meinungen wie sichere und definierte Glaubenssätze der Kirche von jemand in Büchern 
Const. Sollicita, 9 Jul. 1753.) Haec sapientissimi Pontificis verba commemorans Sanctissimus D. Leo XIII ait: «Plena sapientiae ac gravitatis praecepta doctis tradidit ... immo etiam exemplar ad imitandum proposuit Sanctum Thomam Aquinatem, qui pacato semper stilo et gravi dicendi forma utitur, non solum cum docet, veritatemque argumentis communit, sed etiam cum adversarios urget et insectatur. Placet nobis haec eadem Decessoris Nostri praecepta iterum sapientibus commendare, idemque exemplar exhibere ". (Leo XIII, epist. ad Episc. Belgii.) "Pulchrum est, eos, qui catholicum nomen scriptis quotidianis defendunt, prae se ferre amorem veritatis constantem, minimeque timidum; sed simul oportet, nihil eosdem suscipere, quod bono cuiquam viro iure displicet, neque ulla ratione temperantiam deserere, quae cunctarum comes debet esse virtutum. In quo nemo sapiens probaverit, aut stilum vehementem plus quam satis est, aut quidquam vel suspiciose dictum, vel quod temere a personarum obsequio indulgentiaque discedere videatur ". (Leo XIII, epist. ad Episc. Provinc. Taurin., 25 Jan. 1882. Acta et Decreta.)

155. Caritatem ergo in omnibus sectentur ii, qui christiana mente scribere, immo et disceptare volunt; non quidem pavidam illam falsamque caritatem, quae, ut hominibus placeat, Deo displicet, fovet errores, veritatem erubescit, sed eam, quae veritatem simul et pacem diligens, omnia pro veritate, omnia pro iustitia, omnia pro Ecclesia et pro fratrum salute tolerare et libenter subire novit; quae etiam sine superbia de veritate praesumens, sine saevitia pro veritate certans, errantes, sanare, potius vult, quam oppugnare; quae tandem, quantum fieri potest, cum omnibus hominibus pacem habet, quod ut assequatur, de incertis non iudicat, incognita non confirmat, proclivior ausgegeben werden, die Gegenmeinungen aber als Irrtümer verdächtigt. Dadurch werden Unruhen in der Kirche geweckt, Spaltungen zwischen den Lehrern herbeigeführt oder gefördert, und sehr oft die Bande der christlichen Liebe zerrissen“ (Konstit. „Sollicita“ 9. Juli 1753). Indem er diese Worte des sehr weisen Papstes in Erinnerung brachte, sagte Seine Heiligkeit Leo XIII.: „Weise und ernste Gebote hat er den Gelehrten gegeben ... und ihnen sogar den hl. Thomas von Aquin als Vorbild zur Nachahmung hingestellt; denn dieser bedient sich immer einer friedlichen Sprache und einer getragenen Redeweise, nicht nur wenn er lehrt und die Wahrheit mit Beweisen absichert, sondern auch, wenn er den Gegnern zusetzt und ihnen nachsetzt. Wir möchten diese Gebote unseres Vorgängers den Weisen von neuem empfehlen und ihnen dasselbe Vorbild darbieten" (Brief an die Bischöfe von Belgien). „Schön ist es, daß die, welche den katholischen Namen in Zeitungen verteidigen, eine beharrliche und keineswegs furchtsame Liebe zur Wahrheit vorzeigen. Gleichzeitig aber dürfen sie nichts unternehmen, was einem guten Manne mit Recht mißfällt, noch auf irgendeine Weise die Mäßigung vermissen lassen, die eine Begleiterin aller Tugenden sein muß. Hierbei wird kein Weiser weder eine Sprache billigen, die heftiger ist als nötig, noch eine Äußerung, die entweder Verdacht erwecken kann, oder grundlos von der Rücksicht auf Personen oder der Nachsicht abzuweichen scheint" (Leo XIII., Brief an die Bischöfe der Turiner Kirchenprovinz vom 25. Januar 1882).

155. Nach Liebe in allen Dingen also sollen die streben, die in christlichem Geiste schreiben oder sogar auch disputieren wollen; freilich nicht nach jener zaghaften und falschen Liebe, die um den Menschen zu gefallen, Gott mißfällt, die Irrtümer fördert und sich der Wahrheit schämt; sondern nach jener, die Wahrheit und Frieden zugleich liebt, und alles für die Wahrheit, alles für die Gerechtigkeit, alles für die Kirche und das Heil der Brüder zu ertragen und gern auf sich zu nehmen versteht; die ferner ohne Hochmut auf die Wahrheit pochend, ohne Härte für die Wahrheit streitend die Irrenden eher heilen als sie bekämpfen will; die schließlich, soweit das möglich ist, mit allen 
est ad bene credendum de homine, quam ad male suspicandum, non se mul tum dolet errare, cum bene credit de malo, perniciose autem, cum male sensit forte de bono.

156. Similiter magnam cautelam adhibeant, ne in refutandis malorum calumniis et dicteriis, eorum adiutores fiant, et scandala cognita faciant lectoribus suis, quae alias non cognovissent, et impiorum scriptis eorum animos inficiant, praesertim in is quae ad fidem aut mores spectant; iacula enim quacumque manu emissa facile vulnerant; vulneris sanatio non fit nisi dexteritate medici.

157. In particulari, ephemeridum redactores ne levius rumores quoscumque referant, qui hodie per telegraphum quotidie sparguntur: attendant thelegraphos fere omnes in manu esse inimicorum Ecclesiae, iudeorum etc., et saepissime inservire propagandis falsis nuntiis in odium Ecclesiae, $v$. gr. circa acta et agenda a Sancta Sede, circa valetudinem Romani Pontificis etc. ad lucra iniusta procuranda ex animorum commotione, etc. Similiter cauti sint in repetendis notitiis de factis quibuslibet scandalosis, divortiis, duellis, caedibus, ac saltem fugienda ea omnia, quae imaginationem legentis nimis excitent et non paucis sunt offensioni et periculo.

158. Sciant quoque scriptores catholici, arduum esse munus suum, multum laboris et studii, multum etiam humilitatis et caritatis requiri, ut bene illo fungantur; insuper debere ipsos quidem opiniones diei et multitudinis consulere, minime tamen ab illis abripi, sed despicere et confutare et oppugnare quaecumque periculosa fuerint vero Ecclesiae bono, socictatis, familiae. Sed si arduum istud officium est, quia
Menschen Frieden hält. Um das zu erreichen, urteilt sie nicht über Ungewisses, bestätigt nichts Unbekanntes, ist eher dazu geneigt, gut über den Menschen zu denken als bösartig Verdacht zu hegen; es schmerzt sie nicht viel, zu irren, wenn sie gut vom Bösen denkt, gefährlich aber, wenn sie vielleicht böse vom Guten gedacht hat.

156. In ähnlicher Weise sollen sie große Vorsicht walten lassen, daß sie nicht bei der Bekämpfung der Verleumdungen und den Spottreden der Bösen deren Helfer werden und ihren Lesern Ärgernisse bekannt machen, die sie anderweitig nicht erfahren hätten, und ihre Seelen nicht mit den Schriften der Gottlosen vergiften, besonders in den Dingen, die den Glauben und die guten Sitten betreffen. Denn leicht verwunden $\mathrm{Ge}-$ schosse, die von irgendeiner Hand geschleudert werden; die Heilung der Wunde aber kommt nur durch die Geschicklichkeit des Arztes zustande.

157. Insbesondere sollen die Redakteure von Zeitungen nicht allzu leichtsinnig alle möglichen Gerüchte wiedergeben, die heutzutage täglich durch den Telegraphen ausgestreut werden; sie mögen beachten, daß fast alle Telegraphen in der $\mathrm{H}$ and der Feinde der Kirche sind, von Juden usw., und sehr oft dazu dienen, falsche Nachrichten zum Schaden der Kirche zu verbreiten, zum Beispiel über Taten und Vorhaben des Heiligen Stuhles, über den Gesundheitszustand des Papstes und so weiter, um ungerechten Gewinn aus der Erregung der Gemüter zu beschaffen und so weiter. Desgleichen sollen sie vorsichtig sein bei der Wiedergabe von Nachrichten über irgendwelche skandalöse Vorgänge, Ehescheidungen, Duelle, Mordtaten, und wenigstens all das zu vermeiden suchen, was die Einbildungskraft des Lesers zu sehr erregt und für nicht wenige zum Anstoß und zur Gefahr wird.

158. Auch sollen sich die katholischen Schriftsteller bewußt sein, daß ihre Aufgabe schwierig ist, daß viel Arbeit und Anstrengung, und auch viel Demut und Liebe erforderlich ist, um dieselbe gut zu erfüllen; weiterhin, daß sie zwar die Meinungen des Tages und der breiten Masse zu Rate ziehen müssen, sich aber keineswegs davon dürfen fortreißen lassen; vielmehr alles geringschätzen, bekämpfen und widerlegen müs- 
aggressionibus et telis, calumniis et persecutionibus malevolorum illos obiicit, ex altera parte nobilissimum, summe meritorium et gloriosum est inter primarios numerari milites Christi, ut Ecclesiae cooperatores ad fidelium salutem et Dei gloriam. Pro corona ipsi subiiciantur verba haec S.P. Leonis XIII in Encyclica Immortale Dei: "Commune omnium catholicorum scriptorum erit propositum: religionem et patriam conservare. Hoc proposito duas res praeclarissimas consecuturi sunt; alteram, ut adiutores sese impertiant Ecclesiae in conservanda propagandaque sapientia christiana; alteram ut beneficio maximo afficiant societatem civilem, cuius, malarum doctrinarum cupiditatum causa, magnopere periclitatur salus ".

159. Ob oculos etiam habeant verba sequentia, quae circa munia scriptorum ephemeridum catholicarum profert S.P. Leo XIII in Encycl. Etsi nos: "Idem omnes in scribendo spectent, quod maxime expedit, constituant iudicio certo et efficiant; nihil ex his rebus praetermittant, quarum utilis atque expetenda cognitio videatur: gravitate et moderatione dicendi retenta, errores et vitia reprehendant, sic tamen ut careat acerbitate reprehensio, personisque parcatur; deinde orationen adhibeant planam atque evidentem, quam facile queat multitudo percipere ». Et, in allocutione ad Catholicarum Ephemeridum Repraesentantes, similia docuit: "Nullo igitur timore, inquit, nullisque minis absterreantur catholici scriptores (qui suorum mercedem laborum ad vitam reservant aeternam) a detegendis, confringendisque per argumenta omnibus erroribus, illis speciatim qui in Syllabo contineri deprehendatur ". Et, cum ab impugnandis talium errorum propagatoribus non abstineri dixisset $\mathrm{S}$. Pontifex, addidit, talem decere catholicorum scriptorum dicendi rationem, " quae nimirum neque nimia aut intempestiva sermonis sen, was immer für das wahre Wohl der Kirche, der Gesellschaft und der Familie gefährlich ist. Aber wenn diese Aufgabe auch schwierig ist, weil sie den Angriffen und Geschossen, den Verleumdungen und den Verfolgungen der Úbelwollenden ausgesetzt sind, so ist es doch andererseits sehr vortrefflich, höchst verdienstlich und ruhmreich, zu den erstrangigen Soldaten Christi gezählt zu werden, als Mitarbeiter der Kirche zum Heil der Gläubigen und zur Ehre Gottes. Als Ehrenkranz mögen ihnen diese Worte des Heiligen Vaters Leo's XIII. in seiner Enzyklika "Immortale Dei“ entgegengehalten werden: „Der gemeinsame Vorsatz aller katholischen Schriftsteller soll sein: die Religion und das Vaterland zu erhalten. Mit diesem Vorsatz werden sie zwei ganz hervorragende Dinge erreichen, das eine, daß sie sich der Kirche als Helfer zur Verfügung stellen bei der Erhaltung und der Verbreitung der christlichen Weisheit; das andere, daß sie der bürgerlichen Gesellschaft eine sehr große Wohltat erweisen, deren Wohl sehr gefährdet ist durch die Begierde und Sucht nach verderblichen Lehren".

159. Auch sollen sie die folgenden Worte vor Augen haben, die der Heilige Vater Leo XIII. in der Enzyklika „Etsi nos" tiber die Aufgaben der katholischen Journalisten ausspricht: ,Alle mögen beim Schreiben das ins Auge fassen, was am meisten zustatten kommt, es mit sicherem Urteil feststellen und verwirklichen; nichts mögen sie von den Dingen auslassen, deren Kenntnis als nützlich und erstrebenswert erscheint. Mit Ernst und Mäßigung in der Redeweise sollen sie die Irrtümer und Laster tadeln, aber so, daß dem Tadel die Bitterkeit fehlt, und daß man die Personen schont; ferner sollen sie eine deutliche und einleuchtende Sprache verwenden, die die Leute leicht verstehen können“. In seiner Ansprache an die Vertreter katholischer Zeitungen hat er ähnliches gelehrt: „Durch keine Furcht und keine Drohungen sollen sich die katholischen Schriftsteller - die den Lohn ihrer Mühen für das ewige Leben aufbewahren - davon abschrecken lassen, alle Irrtümer aufzudekken und sie mit Beweisen zunichte machen, insbesondere jene, die im Syllabus enthalten sind. Und nachdem der Papst gesagt hatte, da $B$ man nicht darauf verzichten soll- 
acerbitate legentium animos offendat, neque partium studio " aut "privatorum commodis communi bono posthabito deserviat. Segnes enim et ignari veritatis defensores potius hanc produnt quam tradunt, et prudentia carnis inimica est Deo ". te, die Verbreiter von solchen Irrtümern anzugreifen, fügte er hinzu, die Redeweise katholischer Schriftsteller solle geziemenderweise so beschaffen sein, „daß sie weder durch zu große oder ungelegene Bitterkeit die Gemüter der Leser verletzt noch unter Hintansetzung des allgemeinen Wohls ,dem Interesse von Parteien" oder "den Vorteilen von Privatpersonen" dient. Denn träge und unkundige Verteidiger der Wahrheit verraten diese eher, als daß sie sie mitteilen, und die Klugheit des Fleisches ist feindlich gegen Gott“.

Übersetzung: P. Amand Reuter, O.M.J.

\section{SUMMARY}

It was at the end of the 19th century, when a few Mexican bishops already realized the mass media's enormous importance. So they devoted to them on their provincial synod at Gualdarajara in 1896-1897 a detailed paper. These bishops thought it to be their aim and pastoral duty that, apart from the multifarious lecture published by church enemies, there should be published good products as well. Particularly they thought of promoting book-literature as well as suitable periodicales. A part from a financial promotion they planned to build up training-centres for journalists and to offer orientation to the writer's work.

\section{RESUME}

A la fin du XIX. siècle, quelques évêques au Mexique reconnurent déjà l'importance énorme des moyens de communication de masse et leur consacrèrent lors de leur synode provincial à Gualdarajara (18961897) une présentation détaillée. lls le considéraient comme leur but proclamé et leur devoir pastoral qu’a côté de la litérature multiple publiée par les adversaires de l'église, des oeuvres litéraires d'un bon niveau soient publiées.

Plus précisément, ils pensaient à l'encouragement de la publication de livres et d'écritures périodiques. Outre une aide financière, il était prévu de créer des centres de formation pour journalistes ainsi que d'orientier les écrivains dans leur travail.

\section{RESUMEN}

Ya a fines del siglo XIX reconocieron algunos obispos en México la enorme significatión de los medios de comunicación de masas y les dedicaron en su Sînodo Provincial en Guadalajara (1896-1897) una detallada ex posición. Ellos consideraron como su meta manifiesta y su obligación pastoral, el que, junto a las numerosas lecturas publicadas por adversarios de la Iglesia, aparecieran en mercado buenas publicaciones. Más exactamente pensaron en la promoción de libros y periódicos adecuados. Junto a una ayuda financiera estaba también previsto crear centros de capacitación para periodistas, asî como también of recer a los escritores orientación en su trabajo. 